\title{
A novel specific grading standard study of auto-segmentation of organs at risk in thorax: subjective-objective-combined grading standard
}

\author{
Yanchen Ying ${ }^{1,2}$, Hao Wang ${ }^{3}$, Hua Chen ${ }^{1}$, Jianfan Cheng ${ }^{1}$, Hengle Gu${ }^{1}$, Yan Shao ${ }^{1}$, Yanhua Duan ${ }^{1}$, Aihui Feng ${ }^{1}$, \\ Wen Feng ${ }^{1}$, Xiaolong Fu' ${ }^{1}$, Hong Quan ${ }^{2}$ and Zhiyong $\mathrm{Xu}^{1 *}$
}

\section{${ }^{*}$ Correspondence:}

xzyong12vip@sina.com

1 Department of Radiation Oncology, Shanghai Chest Hospital, Shanghai Jiao Tong University, Shanghai 200030, China

Full list of author information is available at the end of the article

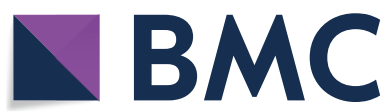

(0) The Author(s) 2021. This article is licensed under a Creative Commons Attribution 4.0 International License, which permits use, sharing, adaptation, distribution and reproduction in any medium or format, as long as you give appropriate credit to the original author(s) and the source, provide a link to the Creative Commons licence, and indicate if changes were made. The images or other third party material in this article are included in the article's Creative Commons licence, unless indicated otherwise in a credit line to the material. If material is not included in the article's Creative Commons licence and your intended use is not permitted by statutory regulation or exceeds the permitted use, you will need to obtain permission directly from the copyright holder. To view a copy of this licence, visit http://creativecommons.org/ licenses/by/4.0/. The Creative Commons Public Domain Dedication waiver (http://creativecommons.org/publicdomain/zero/1.0/) applies to the data made available in this article, unless otherwise stated in a credit line to the data. 
Keywords: SOC grading standard, Auto-segmentation, Thorax, Organs at risk

\section{Background}

Accurate delineation of organs at risk (OARs) is an essential step in ensuring radiotherapy dosimetry accuracy. Over recent years, the auto-segmentation of OARs has gained increasing importance. Compared to cumbersome slice-by-slice manual delineation, auto-segmentation not only saves time to radiation oncologists [1] but also reduces inter- and intra-observer variations [2,3]. A number of commercial auto-segmentation software have been developed and gradually used in clinical, such as MIM Maestro (MIMVista Corp, Cleveland, US-OH), SPICE (Philips, Madison, WI), and ABAS (CMSElekta, Stockholm, Sweden) [4]. However, some studies suggested that the contours generated by auto-segmentation should still be carefully reviewed by radiation oncologists $[3,5-8]$.

At present, the evaluation methods for the auto-segmentation accuracy for thoracic OARs have not yet been standardized. There are three major methods: the geometric objective evaluation method, which includes grading the performance of OAR autosegmentation by geometric indexes. For instance, Velker et al. [9] graded the autosegmentation accuracy into three levels according to Dice similarity coefficient (DSC) as: good $(0.8 \leq \mathrm{DSC} \leq 1)$, medium $(0.6 \leq \mathrm{DSC}<0.8)$, and poor $(0 \leq \mathrm{DSC}<0.6)$. Notably, Ciardo et al. [5] used three indexes of DSC, the difference of the Euclidean distance between centers of mass ( $\triangle \mathrm{CMD}$ ) and average Hausdorff distance (AHD) to grade segmentation accuracy into three levels. This method is quantitative and universal, but it has no support from the subjective evaluation. The second method is the subjective evaluation method $[6,10]$, which grades segmentation accuracy according to the degree of modification required for the auto-segmentation contours that are judged by radiation oncologists' clinical experience and subjective will. This method isn't quantitative; thus, it cannot be popularized to other radiotherapy centers for grading evaluation of the auto-segmentation accuracy. The third method is the subjective and objective combined evaluation method [7]. Recent research in this area obtained the median values of the geometric indexes corresponding to the level of subjective evaluation levels based on DSC and maximum Hausdorff distance (MHD); however, they did not provide the ranges of geometric indexes; thus, this approach cannot be used as a general evaluation standard.

The above three evaluation methods have their own disadvantages. Each one has a different evaluation base, so that it is difficult to horizontally compare the auto-segmentation accuracy between different software for different OARs. Hence, it is extremely urgent to develop a uniform evaluation standard for the accuracy of auto-segmentation software. In addition, a correlation between OAR volume and geometric indexes such as DSC has been previously reported [11]. A specific grading standard for auto-segmentation accuracy is developed for each OAR, thus making research results more accurate.

The auto-segmentation algorithm has been extensively studied for regions such as the head and neck [11-13], and abdomen [14]. Considering thorax, there are also many studies reporting auto-segmentation for common OARs such as heart, lung, spinal cord, trachea, and esophagus [15-18]. Nevertheless, none of these studies have been undertaken for all the thoracic OARs listed in the Radiation Therapy Oncology Group (RTOG) 
delineation guidelines [19], including great vessels, chest wall, and skin. In order to make the content more comprehensive, we tried to study all the thoracic OARs listed in RTOG guidelines.

The aim of this study was to establish an OAR-specific subjective-objective-combined (SOC) grading standard in thorax for evaluating the accuracy of all commercial and selfdeveloped auto-segmentation software. Thirteen thoracic OARs were auto-segmented, and five geometric indexes of DSC, $\triangle \mathrm{CMD}$, the difference of volume $(\Delta \mathrm{V}), \mathrm{MHD}$, and AHD were calculated in our work. The novel OAR-specific SOC grading standard was developed by combining the subjective evaluation standard proposed by us and the geometric objective indexes. We clarified the correspondences between the subjective evaluation levels and the ranges of DSC for thoracic OARs. Consequently, the SOC grading standard should have great potential for applications in the accuracy evaluation of autosegmentation software based on traditional algorithms and deep learning algorithms.

\section{Results}

\section{Grading results by geometric indexes}

Table 1 shows the results of the five geometric indexes between manual and auto-segmentation contours for 13 OARs in the thorax. Table 2 shows the grading results of 13 OARs in the thorax by the geometric objective evaluation method of our center and the other two centers. According to the DSC, we graded the right lung (R Lung), left lung (L Lung), skin, heart, and spinal cord (SC) as Level 3 (mean DSC: 0.88-0.96), which required some manual modification after auto-segmentation; the aorta (AOR), chest wall $(\mathrm{CW})$, trachea, and pulmonary artery (PA) were Level 2 (mean DSC: 0.73-0.79), which required many manual modifications after auto-segmentation; the superior vena cava (SVC), esophagus (ESO), inferior vena cava (IVC), and pulmonary vein (PV) were Level 1 (mean DSC: 0.53-0.62), for which the use of auto-segmentation was not recommended. Compared with Velker et al. [9] and Ciardo et al. [5], the grading results of all the OARs were consistent except for the SVC. Moreover, the grading results of the AHD

Table 1 Five geometric indexes between manual contours and auto-segmentation contours for 13 organs at risk in thorax (mean \pm SD)

\begin{tabular}{lllcll}
\hline Structure & DSC & $\boldsymbol{\Delta C M D}(\mathbf{c m})$ & $\boldsymbol{\Delta V}(\%)$ & $\mathbf{M H D}(\mathbf{c m})$ & $\mathbf{A H D}(\mathbf{c m})$ \\
\hline R Lung & $0.96 \pm 0.02$ & $0.13 \pm 0.07$ & $7 \pm 5$ & $2.18 \pm 0.72$ & $0.11 \pm 0.05$ \\
L Lung & $0.94 \pm 0.03$ & $0.28 \pm 0.28$ & $10 \pm 8$ & $3.45 \pm 2.19$ & $0.17 \pm 0.12$ \\
Skin & $0.93 \pm 0.06$ & $2.06 \pm 2.38$ & $11 \pm 10$ & $8.85 \pm 4.21$ & $0.58 \pm 0.54$ \\
Heart & $0.90 \pm 0.07$ & $0.40 \pm 0.40$ & $7 \pm 10$ & $1.85 \pm 1.11$ & $0.24 \pm 0.17$ \\
SC & $0.88 \pm 0.04$ & $1.34 \pm 2.01$ & $9 \pm 7$ & $2.74 \pm 4.19$ & $0.13 \pm 0.21$ \\
AOR & $0.79 \pm 0.10$ & $0.92 \pm 0.48$ & $24 \pm 20$ & $2.72 \pm 1.15$ & $0.28 \pm 0.16$ \\
CW & $0.77 \pm 0.05$ & $1.29 \pm 0.66$ & $39 \pm 26$ & $6.51 \pm 1.97$ & $0.46 \pm 0.19$ \\
Trachea & $0.75 \pm 0.09$ & $0.53 \pm 0.31$ & $34 \pm 51$ & $4.27 \pm 4.65$ & $0.25 \pm 0.25$ \\
PA & $0.73 \pm 0.09$ & $0.71 \pm 0.44$ & $16 \pm 10$ & $2.12 \pm 1.14$ & $0.28 \pm 0.17$ \\
SVC & $0.62 \pm 0.09$ & $1.17 \pm 0.52$ & $29 \pm 17$ & $1.87 \pm 0.79$ & $0.33 \pm 0.13$ \\
ESO & $0.57 \pm 0.11$ & $0.89 \pm 0.60$ & $33 \pm 33$ & $2.10 \pm 0.74$ & $0.29 \pm 0.13$ \\
IVC & $0.56 \pm 0.16$ & $0.91 \pm 0.62$ & $30 \pm 25$ & $2.17 \pm 1.07$ & $0.43 \pm 0.24$ \\
PV & $0.53 \pm 0.14$ & $1.00 \pm 0.49$ & $35 \pm 24$ & $2.65 \pm 0.90$ & $0.44 \pm 0.11$ \\
\hline
\end{tabular}


Table 2 Grading results of 13 organs at risk in thorax by five geometric indexes

\begin{tabular}{|c|c|c|c|c|c|c|c|c|c|}
\hline \multirow[t]{2}{*}{ Structure } & \multicolumn{3}{|l|}{ DSC } & \multicolumn{2}{|l|}{$\Delta \mathrm{CMD}$} & \multirow{2}{*}{$\begin{array}{l}\Delta V \\
\text { Our } \\
\text { center }\end{array}$} & \multirow{2}{*}{$\begin{array}{l}\text { MHD } \\
\text { Our } \\
\text { center }\end{array}$} & \multicolumn{2}{|l|}{ AHD } \\
\hline & $\begin{array}{l}\text { Our } \\
\text { center }\end{array}$ & $\begin{array}{l}\text { Velker } \\
\text { et al. } \\
\text { [9] }\end{array}$ & $\begin{array}{l}\text { Ciardo } \\
\text { et al. } \\
\text { [5] }\end{array}$ & $\begin{array}{l}\text { Our } \\
\text { center }\end{array}$ & $\begin{array}{l}\text { Ciardo } \\
\text { et al. } \\
\text { [5] }\end{array}$ & & & $\begin{array}{l}\text { Our } \\
\text { center }\end{array}$ & $\begin{array}{l}\text { Ciardo } \\
\text { et al. } \\
\text { [5] }\end{array}$ \\
\hline R Lung & 3 & 3 & 3 & 3 & 3 & 3 & 2 & 3 & 3 \\
\hline L Lung & 3 & 3 & 3 & 3 & 2 & 3 & 1 & 3 & 2 \\
\hline Skin & 3 & 3 & 3 & 1 & 1 & 2 & 1 & 1 & 1 \\
\hline Heart & 3 & 3 & 3 & 3 & 2 & 3 & 2 & 2 & 2 \\
\hline SC & 3 & 3 & 3 & 1 & 1 & 3 & 1 & 3 & 3 \\
\hline$A O R$ & 2 & 2 & 2 & 2 & 1 & 1 & 1 & 2 & 2 \\
\hline$C W$ & 2 & 2 & 2 & 1 & 1 & 1 & 1 & 1 & 1 \\
\hline Trachea & 2 & 2 & 2 & 2 & 1 & 1 & 1 & 2 & 2 \\
\hline PA & 2 & 2 & 2 & 2 & 1 & 2 & 2 & 2 & 2 \\
\hline SVC & 1 & 2 & 2 & 1 & 1 & 1 & 2 & 2 & 2 \\
\hline ESO & 1 & 1 & 1 & 2 & 1 & 1 & 2 & 2 & 2 \\
\hline IVC & 1 & 1 & 1 & 2 & 1 & 1 & 2 & 1 & 1 \\
\hline PV & 1 & 1 & 1 & 2 & 1 & 1 & 1 & 1 & 1 \\
\hline
\end{tabular}

in our center were basically identical with those of Ciardo et al. [5], except for the L Lung.

In addition, the grading results obtained by the $\triangle \mathrm{CMD}$ were quite different from those of Ciardo et al. [5]. The levels of L Lung, heart, AOR, trachea, PA, ESO, IVC, and PV were higher than those of Ciardo et al. [5]. The other two centers have not studied the grading results of the $\Delta \mathrm{V}$ and MHD, so the results of our two indexes could not be compared with other centers.

Based these results, both the DSC and AHD are suitable to be used as the main geometric indexes. Combined with the results of the third part below, we recommend the DSC as the only geometric objective index for auto-segmentation accuracy evaluation.

\section{Grading results by subjective evaluation standard}

The grading results of 13 OARs in the thorax by the subjective evaluation standard are listed in Table 3. Level 3 OARs were the R Lung, L Lung and spinal cord (the range of average percentage to be modified: $6-10 \%$ ); Level 2 OARs were the skin and heart (the range of average percentage range to be modified: 14-17\%); the other eight OARs were Level 1 (the range of average percentage range to be modified: $31-75 \%$ ).

The above grading results were not wholly consistent with the geometric objective evaluation results. For the skin, heart, AOR, CW, trachea, and PA in all the OARs (46.2\%), the grading results of subjective evaluation standard were one level lower than those of the DSC by our center, which suggests that the geometric index cannot fully evaluate auto-segmentation accuracy.

\section{The SOC grading standard}

The SOC grading standard of 13 OARs in the thorax is shown in Table 4. There were two cases, depending on whether the level distribution of the OAR is single. The first type of OARs was the R Lung, CW, PA, SVC, ESO, IVC, and PV. Since all cases for each of the 
Table 3 Grading results of 13 organs at risk in thorax by the subjective evaluation standard

\begin{tabular}{lccll}
\hline Structure & $\begin{array}{l}\text { Average standard } \\
\text { slice number }\end{array}$ & $\begin{array}{l}\text { Average slice number to } \\
\text { be modified }\end{array}$ & $\begin{array}{l}\text { Average percentage to } \\
\text { be modified (\%) }\end{array}$ & $\begin{array}{l}\text { Subjective } \\
\text { score }\end{array}$ \\
\hline R Lung & 71 & 5 & 6 & 3 \\
L Lung & 71 & 7 & 10 & 3 \\
Skin & 125 & 19 & 14 & 2 \\
Heart & 31 & 6 & 17 & 2 \\
SC & 124 & 11 & 8 & 3 \\
AOR & 62 & 27 & 41 & 1 \\
CW & 71 & 29 & 42 & 1 \\
Trachea & 47 & 15 & 31 & 1 \\
PA & 12 & 7 & 58 & 1 \\
SVC & 22 & 13 & 57 & 1 \\
ESO & 72 & 54 & 74 & 1 \\
IVC & 13 & 10 & 75 & 1 \\
PV & 9 & 7 & - & 1
\end{tabular}

OARs were the same level, only one range of the geometric index corresponding to the level could be determined. For example, the DSC, $\triangle \mathrm{CMD}, \triangle \mathrm{V}, \mathrm{MHD}$ and AHD for the $R$ Lung of Level 3 were $0.93-1,0-0.26 \mathrm{~cm}, 0-14 \%, 0-4.27 \mathrm{~cm}, 0-0.19 \mathrm{~cm}$, respectively.

The second type of OARs was the L Lung, skin, heart, spinal cord, AOR, and trachea, and their level distribution wasn't single. Figure 1 shows the correspondences between the subjective evaluation levels and the DSCs of the six OARs. For the L Lung, spinal cord, and AOR, the DSC ranges corresponding to the partial levels for each OAR did not overlap, which were used as the grading standard; for the other OARs or levels, the DSC ranges corresponding to the different levels overlapped. DSC's mean values still increased with the increase of the subjective evaluation levels, so the mean values were selected as the grading standard. In addition, the correspondences between the other four geometric indexes and the levels of the six OARs were less obvious than those of the DSC. So, the four indexes were not suitable as geometric objective evaluation indexes for the OAR auto-segmentation accuracy.

Although the first type of OARs can use all the five indexes to evaluate segmentation accuracy, the second type of OARs is not suitable for evaluation using all indexes. Combined with the results from the first part, we finally chose the DSC as a component of the evaluation indexes in the SOC grading standard.

\section{Discussion}

Thanks to computer technology advancements, the auto-segmentation software based on traditional algorithms and deep learning for OARs has undergone continuous development $[22,23]$. More time is needed to evaluate the segmentation accuracy, although the software can perform auto-segmentation. At present, some studies have reported on the evaluation methods for the accuracy of auto-segmentation software $[5-7,9,10]$; however, their evaluation bases are different. Therefore, it is especially important to develop a uniform evaluation standard for the software's auto-segmentation accuracy.

In this paper, 13 thoracic OARs were auto-segmented by MIM software. Five geometric indexes of DSC, $\triangle \mathrm{CMD}, \triangle \mathrm{V}, \mathrm{MHD}, \mathrm{AHD}$, and the subjective evaluation level were 


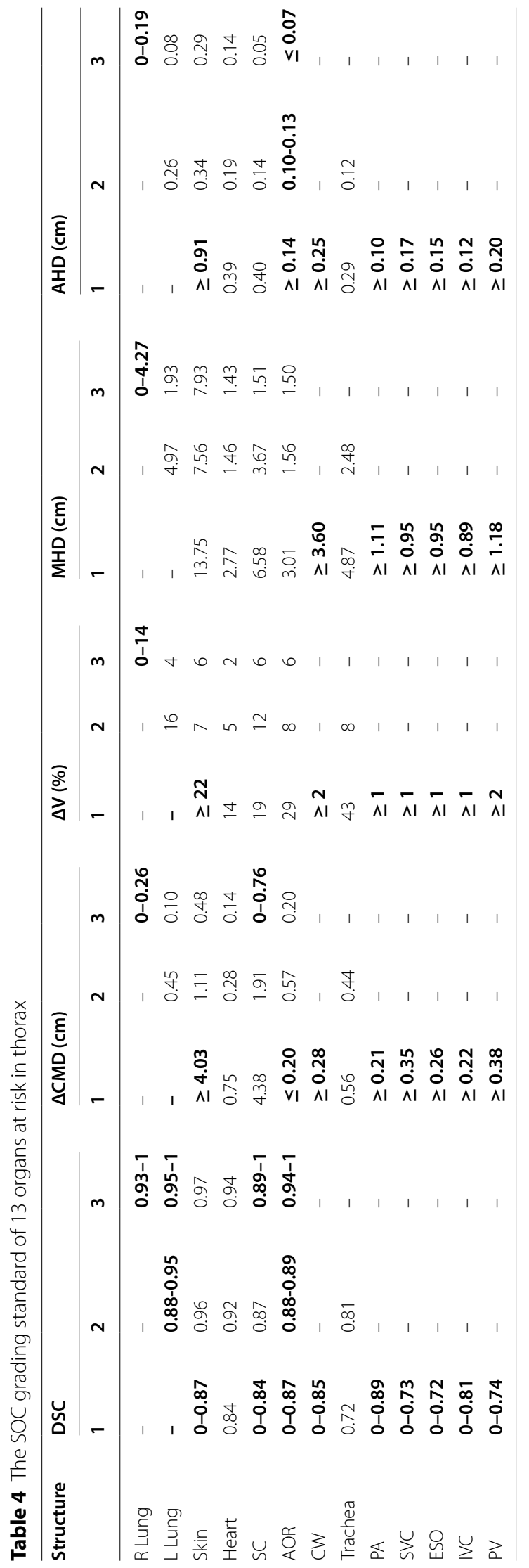




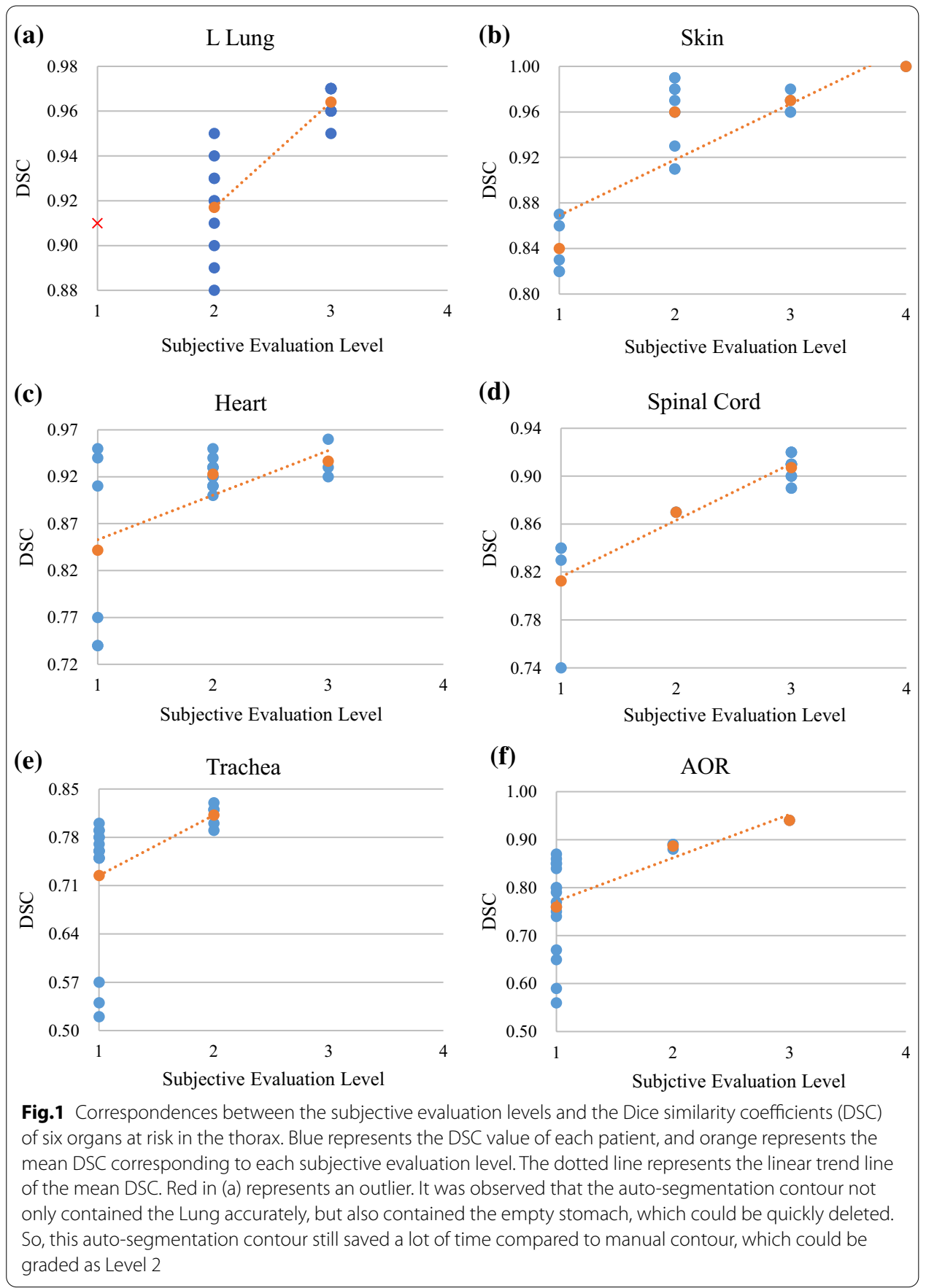

used as the evaluation indexes of auto-segmentation accuracy. This is the first study that proposed an easy-to-operate subjective evaluation standard to the best of our knowledge. In order to improve the consistency of evaluation, we adopted the subjective-objective-combined evaluation method. In this way, the geometric index range corresponding to the subjective evaluation level of each thoracic OAR was found, which was a new OAR-specific SOC grading standard. The SOC grading standard can be used to assess the auto-segmentation accuracy by the value of the geometric index. The standard 
has more clinical universality due to the diversity of thoracic OARs. For other clinical treatment sites, the standard can provide theoretical guidance and research ideas.

The geometric objective evaluation method of auto-segmentation accuracy assesses the difference between auto-segmentation and manual contours according to the geometric index. Five geometric indexes were used in this paper (Table 2). Velker et al. [9] used the DSC, and the grading results obtained by the DSC were consistent with ours. Ciardo et al. [5] used the DSC, $\triangle \mathrm{CMD}$, and AHD. Although their grading results obtained by the DSC and AHD were consistent with ours, their grading results of the $\triangle \mathrm{CMD}$ were quite different from ours. Dolz et al. [24] calculated the $\triangle \mathrm{V}$ and MHD, but they did not grade accuracy according to the two indexes. The papers mentioned above used atlas-based auto-segmentation method which were the same as ours. On the one hand, there are not enough studies that graded segmentation accuracy by the $\Delta \mathrm{V}$ and MHD, thus making it challenging to compare the grading results. On the other hand, the two indexes' grading results were quite different from those of the DSC. Therefore, the two indexes may not be suitable as evaluation indexes, thus suggesting the use of DSC and AHD as the main geometric evaluation indexes. As shown in Table 4, the correspondence between the AHD and the subjective evaluation level is not strong. Hence, the DSC was selected as a component of the evaluation index in the SOC grading standard.

Lustberg et al. [7] reported significant geometric difference between the manual and user-adjusted contour of the esophagus, while both were accepted with local clinical guidelines by the radiation oncologists. Similarly, our results (Tables 2, 3) also showed that the geometric indexes' results were not completely identical to those of subjective evaluation standards. The main reason is that some slices with geometric differences may be subjectively considered do not need to be modified. Therefore, it is likely that the evaluation standard of segmentation accuracy based on geometric indexes alone is not accurate. Morris et al. [6] and Zhu et al. [10] adopted the reliable subjective evaluation method. The radiation oncologist evaluated the auto-segmentation accuracy according to the coincidence degree between the auto-segmentation contours and the anatomical structure of OAR. However, their results cannot be used directly for other centers because of the lack of specific operating procedures.

Based on the above studies, we proposed an easy-to-operate subjective evaluation standard for three different length types of OAR (Table 5). Using this standard, the segmentation accuracy can be directly graded by the number or percentage of CT slice to be modified. The slice numbers of thoracic OARs except for PV (average slices: 10) in this study are more than ten slices. The evaluation standard for the $<3$ slices may apply to small-volume OARs in the head and neck.

Compared with the geometric objective evaluation methods, the subjective evaluation methods by experienced radiotherapists are more reliable. However, the grading results of different radiation oncologists may be different. Especially the grading results of the junior radiation oncologists are more accurate than the senior radiation oncologists. We selected an experienced oncologist as the expert to evaluate the all contours. In this way, inter-observer variations will be reduced, and the consistency of grading results will be improved. The auto-segmentation software for different OARs has different performance. The level distribution for the first type of OARs was single, which included the 


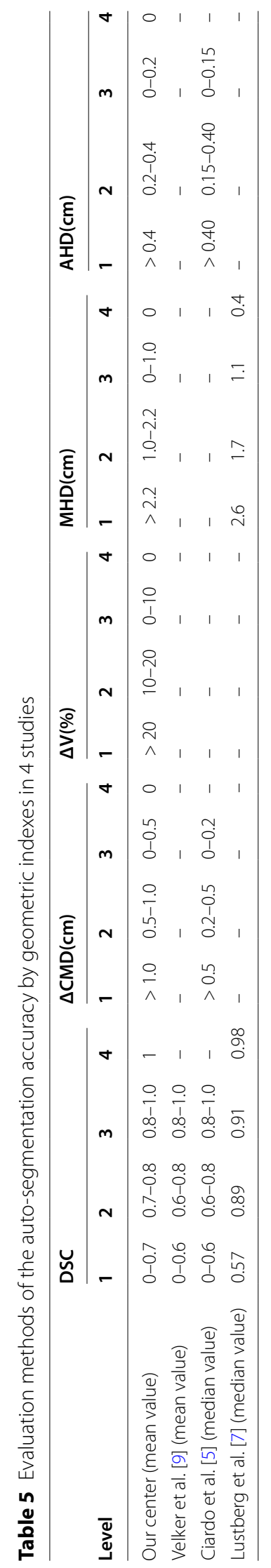


R Lung, CW, PA, SVC, ESO, IVC, and PV. For these OARs, we can only determine the DSC range corresponding to only one level (Table 4). Further investigation is needed to improve their grading standard, except for the R Lung. With the continuous improvement of algorithms and imaging methods, the auto-segmentation accuracy of these poor-performance OARs may be better. The DSC range, which corresponds to higher levels, will be supplemented in the future. In addition, the level distribution of the second OARs was not single, which included the L Lung, skin, heart, spinal cord, AOR, and trachea. The correspondences between their partial, subjective evaluation levels and the DSC ranges were clear and were used for each thoracic OAR to evaluate segmentation accuracy. For the other OARs, the DSC ranges of adjacent levels were overlapped, so the mean values were selected as the grading standard.

It was found that more ranges of the other four geometric indexes except DSC between adjacent levels were not continuous. A possible explanation for this might be insufficient sample. Due to limitations of computed tomography (CT) contrast and resolution, it is even difficult for the radiation oncologist to distinguish where the contours of some OARs should be, such as brachial plexus. It was graded directly as Level 1, which was not recommended for auto-segmentation.

The SOC grading standard proposed in this paper generated preliminary results. The research method is applicable for accuracy evaluation of OARs or tumors auto-segmentation in the thorax. Other radiation centers can directly use the SOC standard for autosegmentation evaluation or obtain their standards by using our method. These grading standards are applicable to the traditional algorithm and auto-segmentation based on deep learning, which is the future developmental direction. On the one hand, these grading standards have great potential to assist radiation oncologists in evaluating the accuracy of OAR auto-segmentation, guiding the clinical use of the auto-segmentation software, and ensuring the accuracy of treatment planning evaluation. On the other hand, these standards might further the resolution of the lack of standardized evaluation methods of auto-segmentation accuracy. It will make the accuracy comparison between different auto-segmentation softwares more meaningful, thereby improving meta-analysis reliability.

\section{Conclusions}

In this work, a novel OAR-specific SOC grading standard in thorax was developed. Compared with the current geometric objective evaluation method and subjective evaluation method, the SOC grading standard represents some improvement in the accuracy evaluation of auto-segmentation software. So, the SOC grading standard provides a possible alternative to evaluating auto-segmentation accuracy based on deep learning and traditional algorithms for thoracic OARs.

\section{Methods}

\section{Patient selection and generation of manual contours}

A total of 40 patients with thoracic malignant tumors treated in our center between November and December 2018, including patients with lung, esophageal, and thymic tumors, were retrospectively selected. The dataset included 12 females and 28 males. The median age of the dataset was 61 years (range 16-78 years). CT scans of each 
patient were obtained by a Siemens Somatom Definition AS CT Scanner System (Siemens Healthcare, Erlangen, Germany). The slice thickness of CT scans was $3 \mathrm{~mm}$. The images were transferred to Pinnacle ${ }^{3}$ treatment planning system (TPS) v9.10 (Philips Healthy, Fitchburg, WI, USA).

Following the RTOG guidelines, a trained radiation oncology resident in our center manually delineated 13 thoracic OARs, including L Lung, right lung, spinal cord, heart, esophagus, chest wall, aorta, pulmonary artery, pulmonary vein, superior vena cava, inferior vena cava, skin and trachea of forty patients on the Pinnacle TPS.

Forty patients were randomly divided into two groups, the training dataset (20) and the test dataset (20). The patients in the two datasets did not intersect. The manual contours of the training dataset were used for training the auto-segmentation algorithm. The manual and auto-segmentation contours of the test dataset were used for evaluating the auto-segmentation accuracy of the algorithm.

\section{Generation of auto-segmentation contours}

Firstly, CT images and structures of the training dataset were transferred to MIM 6.8.7. By using these, we created an atlas library of thoracic OARs for training the auto-segmentation capability of MIM. After the training was completed, the CT images of the test dataset were transferred to MIM. Their auto-segmentation contours were obtained with the simultaneous truth and performance level estimation (STAPLE) algorithm. The manual contours were used as the gold standard to evaluate the auto-segmentation accuracy of thoracic OARs.

\section{Main evaluation methods by geometric indexes}

At present, there are three major methods for evaluating the auto-segmentation accuracy of thoracic OARs: (1) geometric objective evaluation method, (2) subjective evaluation method, and (3) subjective and objective combined evaluation method. As shown in Table 5, the first and third methods involved geometric indexes.

We evaluated the auto-segmentation accuracy of thoracic OARs by five indexes of DSC, $\triangle \mathrm{CMD}, \triangle \mathrm{V}$, MHD, and AHD before, as shown in Table 5. The DSC is defined as

DSC $=\frac{2\left|V_{\text {manual }} \cap V_{\text {attas }}\right|}{\left|V_{\text {manual }} \cap V_{\text {atlas }}\right|}$, where $V_{\text {manual }}$ means the volume of manual contour, and $V_{\text {atlas }}$ means the volume of atlas contour [20]. The range of DSC is $0-1$; where 1 represents the two perfectly coincident contours. The MHD and AHD are the maximum and average distance between two point sets of the two contours, respectively [21]. The smaller the MHD and AHD, the smaller the difference between the two contours.

\section{Main evaluation methods by subjective scoring}

The subjective evaluation method and the subjective and objective combined evaluation method both involved subjective scoring of the auto-segmentation contours by the radiation oncologists listed in Table 6.

We developed an easy-to-operate subjective evaluation standard (Table 7), which divided the auto-segmentation accuracy into four following levels: poor, moderate, good, and great consistency between auto-segmentation and manual contours. Considering that different OARs have different lengths, we discussed three cases according to the CT slices of OAR: $>10$ slices, $3-10$ slices, and $<3$ slices. 
Table 6 Evaluation methods of the auto-segmentation accuracy by subjective scoring in 3 studies

\begin{tabular}{|c|c|c|c|c|c|}
\hline Level & 1 & 2 & 3 & 4 & 5 \\
\hline Morris et al. [6] & $\begin{array}{l}\text { Clinically unac- } \\
\text { ceptable }\end{array}$ & $\begin{array}{l}\text { Major modifica- } \\
\text { tions required }\end{array}$ & $\begin{array}{l}\text { Moderate modifi- } \\
\text { cations required }\end{array}$ & $\begin{array}{l}\text { Minor modifica- } \\
\text { tions required }\end{array}$ & $\begin{array}{l}\text { Clinically } \\
\text { accept- } \\
\text { able }\end{array}$ \\
\hline Zhu et al. [10] & $\begin{array}{l}\text { Useful as autocon- } \\
\text { toured }\end{array}$ & $\begin{array}{l}\text { Useful with minor } \\
\text { edits }\end{array}$ & Not useful & - & - \\
\hline Lustbrg et al. [7] & $\begin{array}{l}\text { No result is useful } \\
\text { basis for further } \\
\text { editing, no time } \\
\text { saving }\end{array}$ & $\begin{array}{l}\text { Some results are } \\
\text { useful for further } \\
\text { editing, little } \\
\text { time saving }\end{array}$ & $\begin{array}{l}\text { Many results are } \\
\text { useful for further } \\
\text { editing, a moder- } \\
\text { ate time saving }\end{array}$ & $\begin{array}{l}\text { Most results are } \\
\text { useful for further } \\
\text { editing, a signifi- } \\
\text { cant time saving }\end{array}$ & - \\
\hline
\end{tabular}

Table 7 Easy-to-operate subjective evaluation standard of the auto-segmentation accuracy

\begin{tabular}{|c|c|c|c|c|}
\hline Level & $\begin{array}{l}\text { Auto-segmentation } \\
\text { performance }\end{array}$ & $\begin{array}{l}>10 \text { slices } \\
\text { (percentage to be } \\
\text { modified) }\end{array}$ & $\begin{array}{l}3-10 \text { slices (slice } \\
\text { number to be } \\
\text { modified) }\end{array}$ & $\begin{array}{l}<3 \text { slices (slice } \\
\text { number to be } \\
\text { modified) }\end{array}$ \\
\hline 1 & $\begin{array}{l}\text { Auto-segmentation is not recom- } \\
\text { mended }\end{array}$ & $20 \%-100 \%$ & $>3$ & 3 \\
\hline 2 & $\begin{array}{l}\text { Many manual modifications are } \\
\text { required after auto-segmentation }\end{array}$ & $10 \%-20 \%$ & $2-3$ & 2 \\
\hline 3 & $\begin{array}{l}\text { Some manual modifications are } \\
\text { required after auto-segmentation }\end{array}$ & $0-10 \%$ & 1 & 1 \\
\hline 4 & $\begin{array}{l}\text { Auto-segmentation can completely } \\
\text { replace manual delineation }\end{array}$ & 0 & 0 & 0 \\
\hline
\end{tabular}

\section{The development of SOC grading standard}

Based on the above subjective evaluation standard, a new SOC grading standard was proposed to overcome the shortcomings of the lack of clinician review of the geometric objective evaluation method and inter-observer variations of the subjective evaluation method. The SOC grading standard was determined by combining the subjective evaluation level of each OAR with the five geometric index ranges corresponding to the level.

The procedure of developing the SOC grading standard was as follows: first, the five geometric index values between the auto-segmentation contours and the manual contours in the test dataset were calculated. Next, an expert radiation oncologist carefully reviewed all the auto-segmentation contours slice by slice and recorded the slice number required modification. Then, all the auto-segmentation contours were graded according to the above subjective evaluation standard. Finally, the range or mean value of the geometric index corresponding to each subjective evaluation level of each OAR was observed, which represented the grading standard of auto-segmentation accuracy.

\footnotetext{
Abbreviations

OAR: Organ at risk; DSC: Dice similarity coefficient; $\triangle \mathrm{CMD}$ : The difference of the Euclidean distance between centers of mass; AHD: Average Hausdorff distance; MHD: Maximum Hausdorff distance; RTOG: Radiation therapy oncology group; SOC: Subjective-objective-combined; $\triangle \mathrm{V}$ : The difference of volume; R Lung: Right lung; L Lung: Left lung; SC: Spinal cord; AOR: Aorta; CW: Chest wall; PA: Pulmonary artery; SVC: Superior vena cava; ESO: Esophagus; IVC: Inferior vena cava; PV: Pulmonary vein; CT: Computed tomography; TPS: Treatment planning system; STAPLE: Simultaneous truth and performance level estimation.
}

\section{Acknowledgements}




\section{Authors' contributions}

Conceptualization - ZX and YY; methodology - HW and HC; formal analysis—JC, HG and AF; data curation—YY, YS and YD; writing-original draft preparation-YY; writing-review and editing - ZX and $\mathrm{HC}$; supervision - XF, WF and $\mathrm{HQ}$; project administration -ZX. All authors read and approved the final manuscript.

\section{Funding}

This work was supported by Nurture projects for basic research of Shanghai Chest Hospital (2019YNJCM05) and the Zhejiang Provincial Natural Science Foundation of China (LSY19H180002).

\section{Availability of data and materials}

The datasets used and/or analyzed during the current study are available from the corresponding author on reasonable request.

\section{Declarations}

Ethics approval and consent to participate

Not applicable.

\section{Consent for publication}

Not applicable.

\section{Competing interests}

The authors declared no competing interests.

\section{Author details}

${ }^{1}$ Department of Radiation Oncology, Shanghai Chest Hospital, Shanghai Jiao Tong University, Shanghai 200030, China. ${ }^{2}$ Key Laboratory of Artificial Micro- and Nano-Structures of Ministry of Education and Center for Electronic Microscopy and Department of Physics, Wuhan University, Wuhan 430070, China. ${ }^{3}$ Institute of Modern Physics, Fudan University, Shanghai, China.

Received: 31 October 2020 Accepted: 24 May 2021

Published online: 03 June 2021

\section{References}

1. Hu K, Lin A, Young A, et al. Timesavings for contour generation in head and neck IMRT: Multi-institutional experience with an atlas-based segmentation method. Int J Radiat Oncol Biol Phys. 2008;72:S391.

2. van Baardwijk A, Bosmans G, Boersma L, et al. PET-CT-based auto-contouring in non-small-cell lung cancer correlates with pathology and reduces interobserver variability in the delineation of the primary tumor and involved nodal volumes. Int J Radiat Oncol Biol Phys. 2007;68:771-8.

3. Eldesoky AR, Yates ES, Nyeng TB, et al. Internal and external validation of an estro delineation guideline-dependent automated segmentation tool for loco-regional radiation therapy of early breast cancer. Radiother Oncol. 2016;121:424.

4. Sharp G, Fritscher KD, Pekar V, et al. Vision 20/20: perspectives on automated image segmentation for radiotherapy. Med Phys. 2014;41:050902.

5. Ciardo D, Gerardi MA, Vigorito S, et al. Atlas-based segmentation in breast cancer radiotherapy: evaluation of specific and generic-purpose atlases. Breast. 2017;32:44-52.

6. Morris ED, Ghanem Al, Pantelic MV, et al. Cardiac substructure segmentation and dosimetry using a novel hybrid magnetic resonance and computed tomography cardiac atlas. Int J Radiat Oncol Biol Phys. 2019;103:985-93.

7. Lustberg T, van Soest J, Gooding M, et al. Clinical evaluation of atlas and deep learning based automatic contouring for lung cancer. Radiother Oncol. 2018;126:312-7.

8. Walker GV, Awan M, Tao R, et al. Prospective randomized double-blind study of atlas-based organ-at-risk autosegmentation-assisted radiation planning in head and neck cancer. Radiother Oncol. 2014;112:321-5.

9. Velker VM. Creation of RTOG compliant patient CT-atlases for automated atlas based contouring of local regional breast and high-risk prostate cancers. Radiat Oncol. 2013;8:188.

10. Zhu M, Bzdusek K, Brink C, et al. Multi-institutional quantitative evaluation and clinical validation of smart probabilistic image contouring engine (spice) autosegmentation of target structures and normal tissues on computer tomog raphy images in the head and neck, thorax, liver, and male pelvis areas. Int J Radiat Oncol Biol Phys. 2013:87:809-16.

11. Isambert A, Dhermain F, Bidault F, et al. Evaluation of an atlas-based automatic segmentation software for the delineation of brain organs at risk in a radiation therapy clinical context. Radiother Oncol. 2008;87:93-9.

12. Daisne JF. Atlas-based automatic segmentation of head and neck organs at risk and nodal target volumes: a clinical validation. Radiat Oncol. 2013:8:154

13. Dean JA, Welsh LC, McQuaid D, et al. Assessment of fully-automated atlas-based segmentation of novel oral mucosal surface organ-at-risk. Radiother Oncol. 2016;119:166-71.

14. Hu P, Wu F, Peng J, et al. Automatic 3d liver segmentation based on deep learning and globally optimized surface evolution. Phys Med Biol. 2016;61:8676.

15. Pirozzi S, Horvat M, Piper J, et al. Atlas-based segmentation: evaluation of a multi-atlas approach for lung cancer. Med Phys. 2012;39:3677

16. Kaderka R, Gillespie EF, Mundt RC, et al. Geometric and dosimetric evaluation of atlas based auto-segmentation of cardiac structures in breast cancer patients. Radiother Oncol. 2019;131:215-20. 
17. Kim J, Han J, Ailawadi S, et al. Multi-atlas based automatic organ segmentation for lung radiotherapy planning. Med Phys. 2016;43:3433.

18. Eduard S, Marcus DM, Tim F. Multi atlas segmentation of thoracic and abdominal anatomy with level set-based local search. J Appl Clin Med Phys. 2014;15:22-38.

19. Kong FM, Quint LMM, Bradley J. Atlas for organs at risk (oars) in thoracic radiation therapy. 2012. https://www.rtog org/CoreLab/ContouringAtlases/LungAtlas.aspx. Accessed 19 July 2019.

20. Dice LR. Measures of the amount of ecologic association between species. Ecology. 1945;26:297-302.

21. Sim DG, Kwon OK, Park RH. Object matching algorithms using robust Hausdorff distance measures. IEEE T Image Process. 1999;8:425-9.

22. Mak RH, Endres MG, Paik JH, et al. Use of crowd innovation to develop an artificial intelligence-based solution for radiation therapy targeting. JAMA Oncol. 2019:5:654-61.

23. Iglesias JE, Sabuncu MR. Multi-atlas segmentation of biomedical images: a survey. Med Image Anal. 2015;24:205-19.

24. Dolz J, Kirişli HA, Fechter T, et al. Interactive contour delineation of organs at risk in radiotherapy: clinical evaluation on NSCLC patients. Med Phys. 2016;43:2569-80.

\section{Publisher's Note}

Springer Nature remains neutral with regard to jurisdictional claims in published maps and institutional affiliations.

- fast, convenient online submission

- thorough peer review by experienced researchers in your field

- rapid publication on acceptance

- support for research data, including large and complex data types

- gold Open Access which fosters wider collaboration and increased citations

- maximum visibility for your research: over 100M website views per year

At BMC, research is always in progress.

Learn more biomedcentral.com/submissions 